\title{
Non-classical Signature of Parametric Fluorescence and its Application in Metrology
}

\author{
M. Hamar ${ }^{1,2}$, V. Michálek, ${ }^{1,2}$ A. Pathak ${ }^{3,4}$ \\ ${ }^{1}$ Institute of Physics of Academy of Sciences of the Czech Republic, Joint Laboratory of Optics of Palacky University , \\ ${ }^{2}$ Institute of Physics of Academy of Sciences of the Czech Republic, 17. listopadu 12, 77207 Olomouc, Czech Republic \\ martin.hamar@upol.cz,vaclav.michalek@upol.cz \\ ${ }^{3}$ RCPTM, Joint Laboratory of Optics of Palacky University and Institute of Physics of the Academy of Sciences \\ of the Czech Republic, tr. 17. listopadu 50a, Olomouc, 77207, Czech Republic, \\ ${ }^{4}$ Department of Physics, Jaypee Institute of Information Technology, A-10, Sector-62, Noida, UP-201307, India, \\ anirban.pathak@gmail.com
}

\begin{abstract}
The article provides a short theoretical background of what the non-classical light means. We applied the criterion for the existence of non-classical effects derived by C.T. Lee on parametric fluorescence. The criterion was originally derived for the study of two light beams with one mode per beam. We checked if the criterion is still working for two multimode beams of parametric down-conversion through numerical simulations. The theoretical results were tested by measurement of photon number statistics of twin beams emitted by nonlinear BBO crystal pumped by intense femtoseconds UV pulse. We used ICCD camera as the detector of photons in both beams. It appears that the criterion can be used for the measurement of the quantum efficiencies of the ICCD cameras.
\end{abstract}

Keywords: Parametric fluorescence, photon number squeezed light, quantum efficiency.

\section{INTRODUCTION}

$\mathrm{T}_{\mathrm{i}}$ HE CLASSICAL optics provide a powerful tool for investigation of properties of strong electromagnetic field. Specifically, the vectors of electric and magnetic field $\mathbf{E}, \mathbf{B}$ are well defined in the classical regime. Further, the uncertainties of the electromagnetic field vectors are mainly caused by the errors of measuring devices. However, in the small energy limit, the nature of light starts to become fuzzy. To be precise, values of the physical observables associated with an electromagnetic wave are no more deterministic and cannot be defined precisely. For example, the energy of light is uncertain and has to be characterized by the statistical formalism of quantum optics. The blurring nature of quantum optics defines the lowest limit of measurement accuracy. On the other hand, quantum optics introduces non-classical states of light that are able to significantly suppress the fluctuations of the quantum noise. For this reason the non-classical light is frequently used in spectroscopy [1], interferometry [2], precision measurement [3], light-wave communications [4], [5], [6], visual science [7] and correlated photon radiometry [8], [9], [10], [11], [12]. The non-classical states have no counterparts in classical optics. The manifestation of non-classical light is mainly twofold, the squeezing [4] and non-classical photon statistics [13]. The non-classical state of light can be generated by a nonlinear optical phenomenon known as spontaneous parametric down-conversion, which is also referred to as parametric fluorescence [14], [15]. Detection of squeezed light is generally very difficult and requires homodyne detection [16]. The detection techniques such as photon number resolving detectors, using the fiber delay loops [17], or CCD cameras with image intensifiers are also used for detection of squeezed light [18], [19], [20]. Many operational criteria that are able to distinguish the classical and non-classical light were derived [21], [22], [15]. Most of them consider only one mode of the field [23] that is occupied mostly by one photon. A few of them were derived for two-modes single photon [24] and multi photon [25], field. We are interested in a specific criterion derived by Lee [26] for multimode and multi photon field. The criterion is used here to investigate the non-classicality of multimode parametric fluorescence detected by CCD cameras with image intensifier. The specific attention is given to the higher-order squeezing [28]. Further, the non-classical nature of the measured data is utilized here to measure the quantum efficiency of the camera.

\section{SUBJECT \& METHODS}

Quantum optics distinguishes two principal states of light, the number state $|n\rangle$ and the coherent state $|\alpha\rangle$. The number state $|n\rangle$, also called the Fock state, was established as a consequence of the particle nature of light. This state reflects the experimental fact that the energy of the light can be changed only by multiples of minimum energy packet photon, $E_{n} \sim n h v$, where $n=1,2,3 \ldots, h$ is the Planck's constant and $v$ is the frequency of the electromagnetic field. Each mode of the electromagnetic field in number state is interpreted as harmonic oscillator which can be nicely described by quantum mechanics. The experiment like the blackbody radiation or photoelectric effect points us to this state of light.

The coherent state $|\alpha\rangle$ was established by the quantum formalism applied to the radiation emitted by a classical monochromatic current. The electric field $\mathbf{E}(\mathbf{r}, t)$ polarized in $x$-direction of light in coherent state is defined by the formula [29] 


$$
\left\langle\mathrm{E}_{x}(\mathrm{r}, t)\right\rangle=2|\alpha|\left(\frac{\hbar \omega}{2 \varepsilon_{0} V}\right)^{\frac{1}{2}} \sin (\omega t-\mathrm{kr}-\theta) .
$$

The full description of coherent state is set by a complex number [29]

$$
\alpha=|\alpha| e^{i \theta}=\langle X\rangle+i\langle P\rangle
$$

where $|\alpha|$ is related to the amplitude of the field, $\theta$ is closed to initial phase of the field, $X$ and $P$ are the quadratures of the field. The quadratures correspond to electric fields $\left\langle\mathrm{E}_{\mathrm{x}}\left(\mathbf{r}, t_{1}\right)\right\rangle,\left\langle\mathrm{E}_{\mathrm{x}}\left(\mathbf{r}, t_{2}\right)\right\rangle$ measured with time delay of quarter period. In quantum optics the standard deviations $\sigma_{x}$ and $\sigma_{p}$ of the quadratures $X$ and $P$ are coupled by the uncertainty principle [30]

$$
\sigma_{x} \sigma_{p} \geq \frac{1}{4}
$$

The standard deviations $\sigma_{x}, \sigma_{p}$ of coherent state are equal $\sigma_{x}=\sigma_{p}=1 / 2$ and fulfill the lowest limit of this inequality, $\sigma_{x} \sigma_{p}=1 / 4$. The quadratures of coherent state can be plotted in phase-space diagram. The variables $X$ and $P$ do not commute and so the coherent state of the light cannot be plotted as a single point in phase space, but as blot parameterized by the distribution function. Mathematical formalism of quantum mechanics identifies three ways to calculate the distribution functions of quadratures of coherent state. The first one computes the GlauberSudarshan P-function, the second one Q or Husimi function and the third one is the Wigner function. The electric field $\mathbf{E}(\mathbf{r}, t)$ is given by the projection of the Wigner function into the $X$-axis of the phase space diagram [29]. Due to blurred distribution function of coherent state, the value of the electric field $\mathbf{E}(\mathbf{r}, t)$ is uncertain. The rate of fluctuations of the field is set by the standard deviation $\sigma_{E}=\left(\hbar \omega / 2 \varepsilon_{0} V\right)^{1 / 2} \sim \sigma_{x}$. The energy of coherent state is uncertain, too. The probability of finding $n$ photons in the field is described by the Poisson function [29]

$$
P(n)=e^{-|\alpha|^{2}} \frac{|\alpha|^{2 n}}{n !}=e^{-\langle n\rangle} \frac{\langle n\rangle^{n}}{n !},
$$

where $|\alpha|^{2}$ corresponds to the average photon number $\langle n\rangle$ of the field. The width of the energy distribution function causes the nonzero standard deviation of photon number, $\sigma_{n} \approx \sqrt{\langle n\rangle}$, what is demonstrated by shot-noise in optical detection.

In spite of the fact that calculation methods of quadrature distribution functions were derived for coherent state, there are no restrictions to calculate all three functions also for the number state or for the sum of number states. Surprisingly for many states, titled as non-classical states, the distribution functions, mainly $\mathrm{P}$ and Wigner function, lost the probabilistic interpretations. The functions may become negative or $\mathrm{P}$ function may start to become more singular than $\delta$ function. The non-classical states do not have counterparts in classical optics. We distinguish three principal states of non-classical light, phase squeezed, amplitude squeezed and photon number squeezed states. The first one has phase uncertainty, the second one has the amplitude uncertainty smaller than coherent state. Last two states have the photon number uncertainties smaller than coherent state, which make them easier to detect. The observation of photodetection noise below the shot-noise limit is thus one of the ways how squeezed states are detected in the laboratory.

The physically simplest detection technique of subPoissonian light is the counting of photons per time period $\Delta t$. We set the average number $\langle n\rangle$ and the standard deviation $\sigma_{n}$ of photons detected per period $\Delta t$, and then we calculate the Fano factor $F$

$$
F=\frac{\operatorname{var}(n)}{\langle n\rangle}=\frac{\sigma_{n}^{2}}{\langle n\rangle}
$$

Fano factor is equal to one $(F=1)$ for coherent state, less than one $(F<1)$ for sub-Poissonian light and greater than one for super-Poissonian light $(F>1)$. This criterion can be applied only for photon statistics of single-mode radiations [26]. It was shown by Agarwal [27] that the two-mode field of coherent state is able to exhibit non-classical signature of its statistics in spite of the fact that Fano factor is 1 or higher. This effect is called the intramode antibunching. For these reasons Lee derived the criterion that concerns the non-classicality among many modes occupied by many photons [26]. Unfortunately, the criterion requires the field detection of each mode by individual sensor. For this reason we decided to take the simplest version of this criterion that concerns only two modes $a, b$ of the fields that are occupied by many photons [25]

$$
R_{a b}(l, m)=\frac{\left\langle n_{a}^{(l+1)} n_{b}^{(m-1)}\right\rangle+\left\langle n_{a}^{(m-1)} n_{b}^{(l+1)}\right\rangle}{\left\langle n_{a}^{(l)} n_{b}^{(m)}\right\rangle+\left\langle n_{a}^{(m)} n_{b}^{(l)}\right\rangle}-1<0,
$$

where $n_{x}^{(l)}=n_{x} ! /\left(n_{x}-l\right) !, x=a, b$ and $l>m$ is the order of non-classicality and $\left\langle n_{a}^{(k)} n_{b}^{(l)}\right\rangle$ is the two-mode factorial moment, which is proportional to the probability of simultaneous detection of $l$ photon in $b$ mode and $k$ photon in $a$ mode. Specifically,

$$
\left\langle n_{a}^{(k)} n_{b}^{(l)}\right\rangle=\sum_{n_{a}} \sum_{n_{b}} n_{a}^{(k)} n_{b}^{(l)} P\left(n_{a} n_{b}\right),
$$

where $P\left(n_{a}, n_{b}\right)$ is the joint distribution function of two modes $a, b$, while each mode is detected separately. The negative value of $R_{a b}(l, m)$ is the sign of non-classicality of the detected light. For this reason we introduce the title - the non-classical signature - that is always associated with the negative value of Lee's factor, $R_{a b}(l, m)<0$, of detected light. 
The rate of correlations between photon numbers of two beams may be expressed as

$$
G_{12}=\frac{\left\langle n_{1} n_{2}\right\rangle}{\left\langle n_{1}\right\rangle\left\langle n_{2}\right\rangle}=\frac{\sigma\left(n_{1}, n_{2}\right)}{\left\langle n_{1}\right\rangle\left\langle n_{2}\right\rangle}+1,
$$

where $\sigma\left(n_{1}, n_{2}\right)=\left\langle n_{1} n_{2}\right\rangle-\left\langle n_{1}\right\rangle\left\langle n_{2}\right\rangle$ is the covariance of photon numbers of modes 1 and 2 , and $\left\langle n_{k}\right\rangle$ is the average photon number in the $k$-th mode (here $k=1,2$ ). The ratio $G_{12}=1$ implies the absence of any correlation in photon numbers, and $G_{12}>1$ implies the existence of an inter-beam correlation in photon numbers.

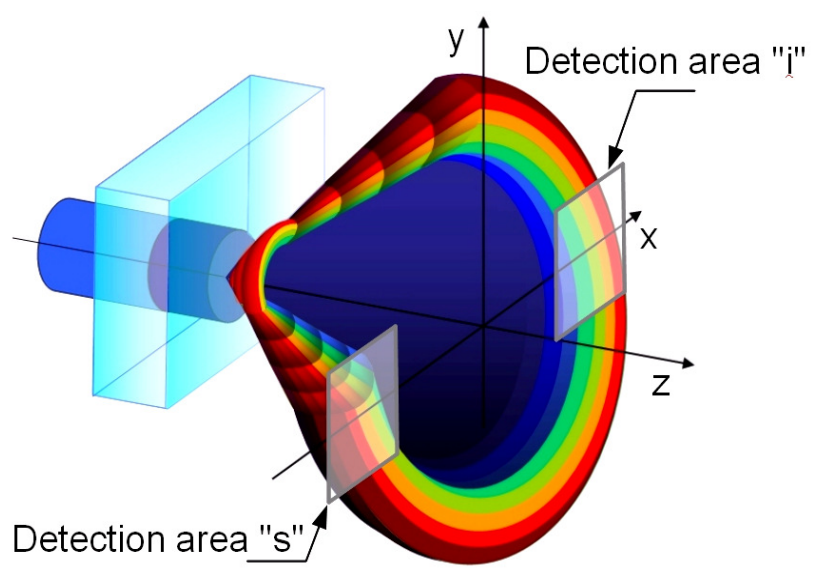

Fig.1. The region of incidence of photon pairs generated by parametric fluorescence is well approximated by the cone behind the crystal. The rectangles indicate the position of detectors.

\section{RESULTS}

In the present work we apply the criterion (6) for measurement of the multimode field detected by the two detectors. The letters $a, b$ in (6), (7) are used here to distinguish the detectors in the rest of the paper. Question is, whether the criterion also works in this case. Firstly, we have investigated this problem by numerical simulations and then we have used this criterion to assess the nonclassicality of real experimental data.

At the beginning, we applied the criterion (6) to assess the non-classical light generated by multimode parametric fluorescence [14] and detected by two detectors, Fig.1. The photon of the pump beam is split spontaneously into two photons with lower energy inside the nonlinear crystal. The photon pairs are correlated in momentum [31] and in the space. The coordinates of detection of signal and idler photons are centrally symmetric in a coordinate system with the $z$-axis being identical to the pump beam axis, Fig.1. The photon pairs which leave the crystal are emitted into space defined by the cone, Fig.1. The top of the cone is placed inside the crystal. The area of photons of the same wavelength is well approximated by the circle, centered on the $z$-axis, in any plane parallel to the output crystal plane. The total numbers of emitted photon pairs in the circular area randomly fluctuate, but the numbers $n_{s}, n_{i}$ of photons emitted into opposite sectors ' $\mathrm{s}$ ' and ' $\mathrm{i}$ ' are correlated, $n_{s}=n_{i}$, Fig.1. The joint distribution function $P_{s i}\left(n_{s}, n_{i}\right)$ of multimode parametric fluorescence measured by two detectors placed in opposite sectors of the cone is [32]

$$
P_{s i}\left(n_{s}, n_{i}\right)=\left\{\begin{array}{c}
P\left(n, M_{s i}\right), n_{s}=n_{i}=n \\
0, n_{s} \neq n_{i}
\end{array},\right.
$$

where $P\left(n, M_{s i}\right)$ is the negative binomial distribution which can be written as

$$
P\left(n, M_{s i}\right)=\left(\begin{array}{c}
M_{s i}+n-1 \\
n
\end{array}\right) \frac{\left(\langle n\rangle / M_{s i}\right)^{n}}{\left(1+\langle n\rangle / M_{s i}\right)^{n+M}},
$$

where $\langle n\rangle$ is the average number of photon pairs incident on the detection areas and $M_{s i}$ is the number of modes of the detected non-classical field.
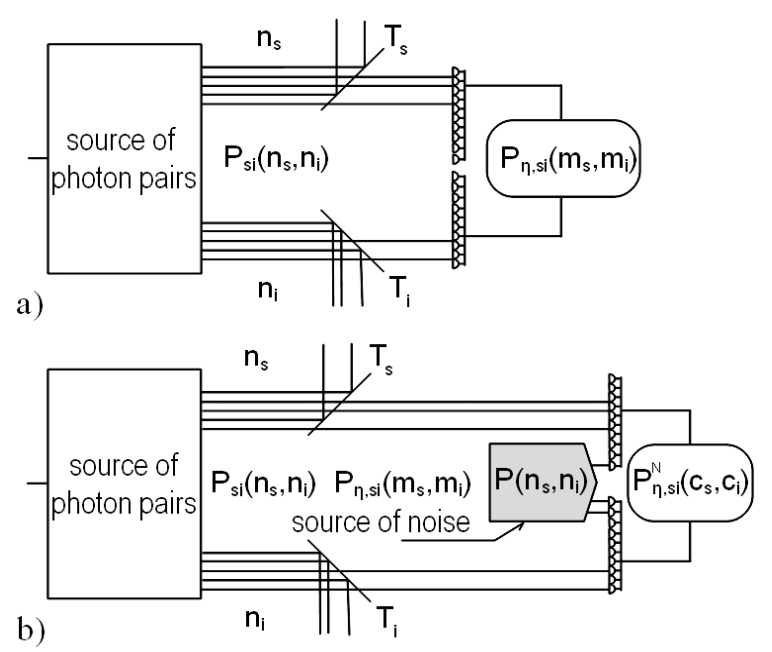

Fig.2. Scheme of the numerical models considered a) the low quantum efficiency of detectors and b) thermal dark-current noise.

In our considerations, the source of classical light is represented by the thermal light generated by two light bulbs. The light of each bulb is detected separately by assigned sensors. The average value of photons incident on detectors is $\left\langle n_{s}\right\rangle,\left\langle n_{i}\right\rangle$. In this case, the number of photons received by each detector is uncorrelated. The sensors detect the field with identical numbers $M_{s}, M_{i}$ of modes $\left(M_{s}=M_{i}=M\right)$. The joint photon number distribution $P\left(n_{s}, n_{i}\right)$ of classical light in this particular case is given by the product of two negative binomial distributions (10)

$$
P\left(n_{s}, n_{i}\right)=P\left(n_{s}, M_{s}\right) P\left(n_{i}, M_{i}\right),
$$

where $\langle n\rangle, M_{s i}$ are substituted by $\left\langle n_{j}\right\rangle, M_{j}, j=s, i$.

The effect of the low quantum efficiency of detectors was simulated by lossless beam splitters with the transmittances $T_{j}, j=s, i$, less than one, placed in front of the detectors, Fig.2.a). The joint distribution function of parametric fluorescence detected by sensors of low quantum efficiencies, $T_{j}<1, j=s, i$, is 


$$
P_{\eta, s i}\left(m_{s}, m_{i}\right)=\sum_{n_{s}=m_{s}}^{\infty} \sum_{n_{i}=m_{i}}^{\infty} \prod_{j=s, i}\left(\begin{array}{l}
n \\
m
\end{array}\right) T^{m_{j}}(1-T)^{n_{j}-m_{j}} P_{s i}\left(n_{s}, n_{i}\right)
$$

where $m_{s}$ and $m_{i}$ are the numbers of signal and idler detection events of detectors generated by detection of $n_{s}$ and $n_{i}$ photons. The joint distribution function $P_{\eta}\left(m_{s}, m_{i}\right)$ of classical light detected by the sensors of low quantum efficiencies is simulated by the same formula (12), where $P_{s i}\left(n_{s}, n_{i}\right)$ is substituted by $P\left(n_{s}, n_{i}\right)(11)$. The detector with quantum efficiency $\eta=100 \%$ is simulated by setting the unit transmittance $T=1$. On the contrary, setting the zero transmittance $T=0$ simulates the detector with zero quantum efficiency $\eta=0 \%$.

Table 1. The statistical parameters accounted for joint distributions $P_{s i}, P$ of non-classical (Fig.3.a)) and classical (Fig.3.b)) light source detected by ideal and real detectors $P_{\eta, s i}, P_{\eta}$ (Fig.3.c), d)) as well as for the resulting joint photon number distribution $P_{s i}^{N}$ (Fig.4.b)) given by simultaneous detection of non-classical and classical sources unaffected and affected $P_{\eta, s i}{ }^{N}$ by losses. The last two columns collect the statistical parameters accounted for the joint photon number distribution $P_{s i}{ }^{\sigma}$ of non-classical light source generated by fluctuated pumping detected by ideal and real $P_{\eta, s i}{ }^{\sigma}$ detector, (Fig.5.a), b)).

\begin{tabular}{|c|c|c|c|c|c|c|c|c|}
\hline & \multicolumn{8}{|c|}{$M_{s i}=M=20, T_{\mathrm{s}}=T_{\mathrm{i}}=T$} \\
\hline & $P_{s i}$ & $P_{\eta, s i}$ & $P$ & $P_{\eta}$ & $\boldsymbol{P}_{s i}^{N}$ & $\boldsymbol{P}_{\eta, s i}{ }^{N}$ & $P_{s i}{ }^{\sigma}$ & $\boldsymbol{P}_{\eta, s i}$ \\
\hline$\langle n\rangle$ & 50 & 50 & 0 & 0 & 50 & 50 & 50 & 50 \\
\hline$\left\langle n_{s}\right\rangle$ & 0 & 0 & 50 & 50 & 50 & 50 & 0 & 0 \\
\hline$\left\langle n_{i}\right\rangle$ & 0 & 0 & 50 & 50 & 50 & 50 & 0 & 0 \\
\hline$\sigma_{r}(\langle n\rangle)$ & $0 \%$ & $0 \%$ & $0 \%$ & $0 \%$ & $0 \%$ & $0 \%$ & $50 \%$ & $50 \%$ \\
\hline$T$ & 1 & 0.2 & 1 & 0.2 & 1 & 0.2 & 1 & 0.2 \\
\hline$\left\langle n_{\eta, s}\right\rangle$ & 50 & 10 & 50 & 10 & 100 & 20 & 51.3 & 10.3 \\
\hline$F_{s}$ & 3.5 & 1.5 & 3.5 & 1.5 & 3.5 & 1.5 & 14.9 & 3.78 \\
\hline$F_{i}$ & 3.5 & 1.5 & 3.5 & 1.5 & 3.5 & 1.5 & 14.9 & 3.78 \\
\hline$R_{a b}(1,1)$ & & 186 & & & & 073 & & 150 \\
\hline$R_{a b}(2,1)$ & & 350 & & & & 144 & & 252 \\
\hline$R_{a b}(2,2)$ & & 168 & & & & 070 & & 111 \\
\hline$R_{a b}(3,1)$ & & 495 & 0.1 & & & 213 & & 329 \\
\hline$R_{a b}(3,2)$ & & 318 & 0.0 & & & 138 & & 196 \\
\hline$R_{a b}(3,3)$ & -0 . & 153 & 0.0 & & & 067 & & 089 \\
\hline$R_{a b}(4,4)$ & & 141 & & & & 064 & & 075 \\
\hline$G_{12}$ & & 700 & & & & 175 & & 900 \\
\hline
\end{tabular}

The distribution of parametric fluorescence $P_{s i}\left(n_{s}, n_{i}\right)$ takes the form of sharp diagonal, Fig.3.a), in contrast to distribution of two uncorrelated thermal sources $P\left(n_{s}, n_{i}\right)$, Fig.3.b). The diagonal elongated shape of the distribution $P_{\eta, s i}\left(m_{s}, m_{i}\right)$ is still visible even when the non-classical light is detected by the sensors with low quantum efficiencies, $\eta_{i}=\eta_{s}=20 \%$, Fig.3.c). Unfortunately, the distribution $P_{\eta, s i}\left(m_{s}, m_{i}\right)$ of non-classical light became blurred for the distribution $P_{\eta}\left(m_{s}, m_{i}\right)$ of thermal sources, Fig.3.d), which makes it harder to identify the non-classicality. Lee's factor $R_{a b}(l, m)$ (6) calculated for distribution of parametric fluorescence, Fig.3.a), is negative for any order $l, m$ unlike the uncorrelated thermal source, Fig.3.b), Table 1. The criterion (6) is satisfied also for any $\langle n\rangle$ and $M_{s i}$ despite reaching $R_{a b}(l, m)$ to zero with increasing of mean number $\langle n\rangle$ of photon pairs and decreasing number of modes $M_{s i}$, Fig.3.e). The non-classical signature of parametric fluorescence is stronger for lower intensities $(\langle n\rangle<2)$ than for higher. The field of multimode parametric fluorescence $\left(M_{s i}>5\right)$ exhibits stronger non-classical signature compared to the single mode fields. This is in accordance with recent observations of Bondani [33]. The field of two uncorrelated balanced, $\left\langle n_{s}\right\rangle=\left\langle n_{i}\right\rangle$, thermal beams has no signature of non-classicality, $R_{a b}(l, m)>0$, for any $\langle n\rangle$ and $M$. Classical field of only a few modes $(M<9)$ per beam exhibits the dependency of $R_{a b}(l, m)$ on the beam intensities $\left\langle n_{s}\right\rangle=\left\langle n_{i}\right\rangle=\langle n\rangle$, Fig.3.f). The factor $R_{a b}(l, m)$ of multimode classical field $(M>9)$ is not affected by its intensity, Fig.3.f). The mentioned character of the factor $R_{a b}(l, m)$ is also satisfied for any order of $l$ and $m$.
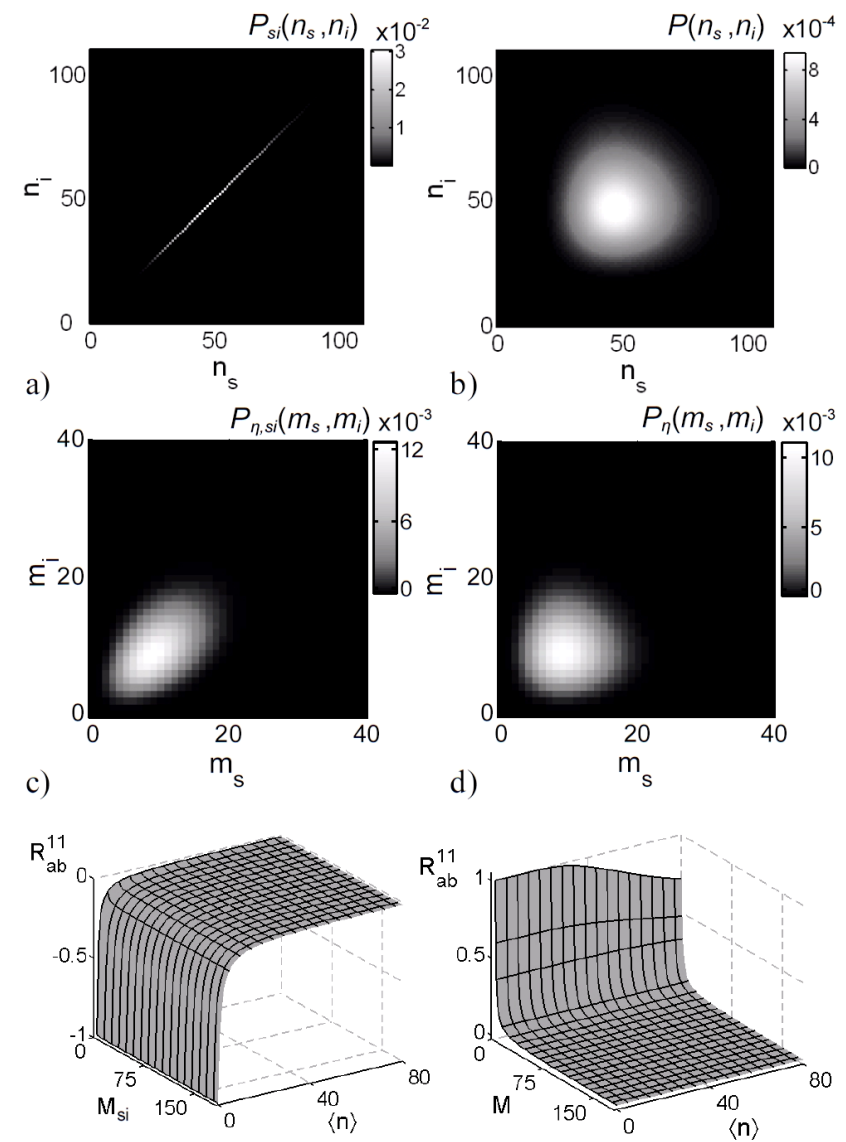

e)

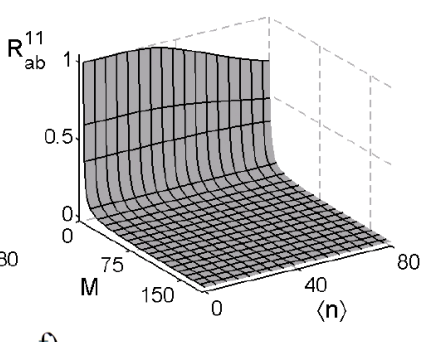

f)

Fig.3. Joint photon number distribution of non-classical a), c) and classical b), d) light source, $\langle n\rangle=\left\langle n_{s}\right\rangle=\left\langle n_{i}\right\rangle=50, M_{s i}=M=20$, accounted for ideal detectors $(T=1)$ a), b) and for real one $(T=0.2) \mathrm{c}), \mathrm{d})$. Lee's factor $R_{a b}(1,1)$ accounted for parametric fluorescence e) and for two thermal sources $f$ ) as a function of intensities of the fields $\langle n\rangle=\left\langle n_{s}\right\rangle=\left\langle n_{i}\right\rangle$ and the number of modes $M_{s i}, M\left(T_{s}=T_{i}=1\right)$.

Interestingly, the signature of the field, no matter if classical or non-classical, is not changed by the quantum efficiencies of the balanced $\left(\eta_{s}=\eta_{i}\right)$ detectors, Table 1 . This is very useful for measurement of quantum efficiencies of 
balanced detectors. The idea is very simple. The light source of known irradiance $I_{e}$ shines at the detector of known area $S$. The energy $E_{d}$ of light detected by the sensor is

$$
E_{d}=\eta I_{e} S=\eta\langle n\rangle,
$$

where $\eta$ is the quantum efficiency of the detector. We expressed the total light energy incident at the detector thorough the average photon number $\langle n\rangle, I_{e} S=\langle E\rangle=\langle n\rangle$. The key parameter is the precise knowledge of the number $\langle n\rangle$. The energy of the light is always detected by the imperfect $(\eta<100 \%)$ detectors, and the precise value of the average photon number is mostly unknown.

This problem can be solved by using non-classical light source. The factor $R_{a b}(l, m)$ of the source is not affected by quantum efficiencies of balanced detectors and can be used for calibration of the light irradiance. The average photon number $\langle n\rangle$ can be derived from the known dependence of the factor $R_{a b}{ }^{l m}(\langle n\rangle, M)$ on $\langle n\rangle$ and $M_{s i}$, Fig.3.e), and by the measurement of the factor $R_{a b}(l, m)$ of non-classical light. The problem is equivalent to solving two equations with two unknowns in mathematics. Unfortunately, the analytical solution is not known and the problem has to be solved numerically, Fig.6.a). The first step of the procedure is to find the curve (dashed green line Fig.6.a)) formed by the intersection of the horizontal plane (orange color shaded plane Fig.6.a)) with 3D surface $R_{a b}^{l m}(\langle n\rangle, M)$ (blue shaded surface Fig.6.a)). The horizontal plane is crossing the $z$-axis of graph Fig.6.a) in value $R_{a b}(l, m)$ given by the measurement of the light used for irradiance calibration. This step has to be repeated separately for each order $l, m$. Next step is to find the projection of the curve (red line Fig.6.a)) formed by the surface's intersection into the $x-y$ plane. The projection curves calculated for different order $l, m$ are intersecting in one point $\left[\langle n\rangle, M_{s i}\right]$, Fig.6.b), with coordinates defined by the average photon number $\langle n\rangle$ and the number $M_{s i}$ of the modes of the investigated non-classical light source. The quantum efficiency $\eta_{j}$ of used detector is calculated by the formula

$$
\eta_{j}=\frac{\left\langle n_{\eta, j}\right\rangle}{\langle n\rangle}, j=s, i
$$

where $\left\langle n_{\eta, j}\right\rangle, j=s, i$ is the average value of photon number detected by an imperfect sensor $\left(T_{j}<1, j=s, i\right)$

$$
\left\langle n_{\eta, j}\right\rangle=\sum_{j} n_{j} \sum_{l} P_{\eta, s i}\left(m_{j}, m_{l}\right), j=s, i, l=i, s .
$$

We obtain $\left\langle n_{\eta, s}\right\rangle=\left\langle n_{\eta, i}\right\rangle$ only for balanced detectors. The classical light cannot be used for calibration by this method, because the factor $R_{a b}(l, m)$ of classical source is independent of the intensity $\langle n\rangle=\left\langle n_{s}\right\rangle=\left\langle n_{i}\right\rangle$, Fig.3.f). The precision of the proposed method of measurement of quantum efficiency is dependent on the measurement errors of the factor
$R_{a b}(l, m)$, which is mostly given by the statistical errors. The statistical errors present in the estimation of the factor $R_{a b}(l, m)$ mostly depend on the size of the statistical ensemble. For ICCD applications, it is the number of images taken. Thus, the higher precision requires a higher number of images, what takes a longer times period over which the mean intensity of the non-classical light has to be carefully stabilized. Generally, the statistical errors present in the factor $R_{a b}(l, m)$ are observed to increase with the orders $l, m$. The proposed method of measurement is designed only for detectors with almost equal quantum efficiencies.

Any discrepancies in quantum efficiencies would lead to a change in the measured non-classical signature and as a consequence the calculated value of quantum efficiency will no longer be the correct one.
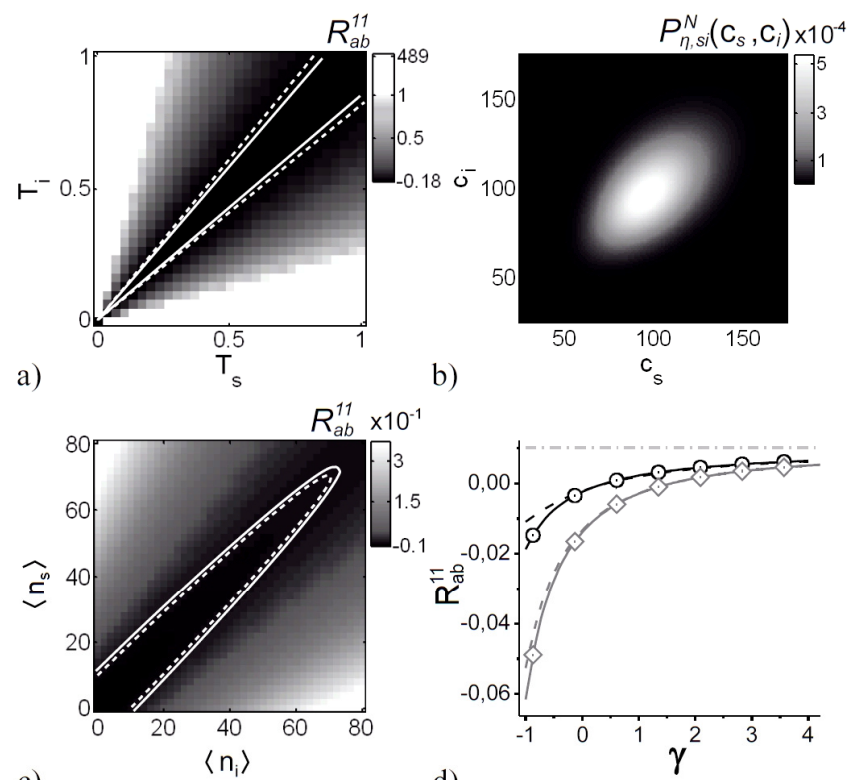

c)
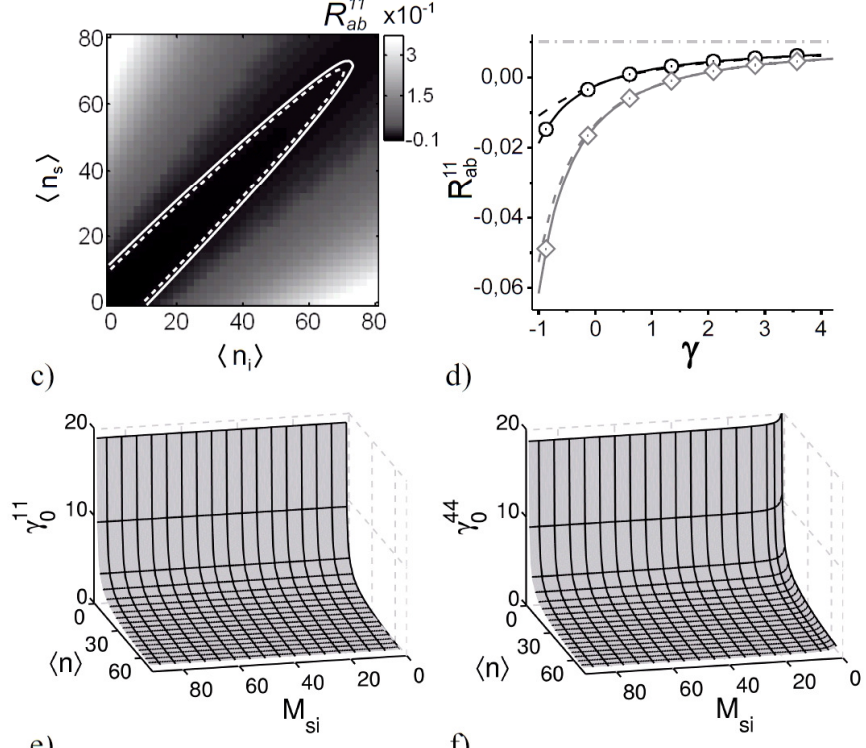

f)

Fig.4. a) The dependence of the factor $R_{a b}(1,1)$ of parametric fluorescence $\left(\langle n\rangle=50, M_{s i}=20\right)$ on transmittances $T_{j}, j=s, i$. b) Joint photon number distribution $P_{\eta, s i}^{N}$ of the mixture of nonclassical (Fig.3.a)) and classical (Fig.3.b)) light. c) The dependence of $R_{a b}(1,1)$ of the mixed non-classical $\left(\langle n\rangle=50, M_{s i}=20\right)$ and classical signals $(M=100)$ on intensities of inhomogeneous noise $\left\langle n_{s}\right\rangle \neq\left\langle n_{i}\right\rangle$. d) The dependence of the factor $R_{, a b}(1,1)$ on the relative intensity $\gamma$ of uniform noise $\left\langle n_{s}\right\rangle=\left\langle n_{i}\right\rangle,(M=100)$. The dependence of the relative threshold intensities of the noise $\left.(M=100) \gamma_{0}{ }^{11} \mathrm{e}\right)$ and $\left.\gamma_{0}^{44} \mathrm{f}\right)$ on the number modes $M_{s i}$ and the intensity $\langle n\rangle$ of the non-classical light. 
The strongly unbalanced detectors are able to completely erase the non-classical signature of the field, Fig.4.a). The factor $R_{a b}(1,1)$ is lower than zero in the area delimited by dashed lines, Fig.4.a). If the difference between the quantum efficiencies $\eta_{s}, \eta_{i}$ of the detectors exceeds the tolerable value (18\% for the case depicted in Fig.4.a)) the nonclassical signature is no longer visible. The tolerated difference is even lower for higher order of Lee's factor $R_{a b}(4,4)$ (solid lines Fig.4.a)).

The non-classical light is mostly contaminated by a noise under experimental conditions. The noise is generated mostly by the extraneous light that reaches the detector (the scattered laser beam) and by the detector itself - the darkcurrent noise (gain noise, circuit thermal noise). In the rest of the paper we consider the dark-current noise as the only source of noise. The photon statistics of the parametric fluorescence affected by the noise contamination is simulated by the convolution of the joint distribution function $P_{\eta, s i}\left(m_{s}, m_{i}\right)$ of detected non-classical light (12) with distribution function $P\left(n_{i}, n_{j}\right)$ of classical thermal light (11), that substitutes the dark-current noise

$$
P_{\eta, s i}^{N}\left(c_{s}, c_{i}\right)=\sum_{m_{s}=0}^{c_{s}} \sum_{m_{i}=0}^{c_{i}} P\left(c_{s}-m_{s}, c_{i}-m_{i}\right) P_{\eta, s i}\left(m_{s}, m_{i}\right)
$$

where $c_{s}$ and $c_{i}$ are the detection events generated by the detection of $n_{s}$ and $n_{i}$ noise photons and $n$ photon pairs. The detection of thermal light is not affected by the quantum efficiencies of the detectors $\left(T_{j}=1, j=s, i\right.$, Fig.2.b)). For simplicity, we consider the balanced detectors $\left(\eta=\eta_{s}=\eta_{i}\right)$ affected by the noise with average photon number $\left\langle n_{j}\right\rangle, j=s, i$, uniformly distributed between the detectors $\left(\left\langle n_{s}\right\rangle=\left\langle n_{i}\right\rangle\right)$. We define the non-classical signal to thermal noise ratio of mixed light sources, according to the relation

$$
\gamma=\frac{\left\langle n_{j}\right\rangle-\left\langle n_{\eta, j}\right\rangle}{\left\langle n_{\eta}\right\rangle}, j=s, i
$$

where $\left\langle n_{\eta, j}\right\rangle$ (15) is the average photon number of detected non-classical light, $\left\langle n_{\eta, s}\right\rangle=\left\langle n_{\eta, i}\right\rangle$. The signal to noise ratio $\gamma$ is equal to minus one when the noise intensity is zero. If the intensities of the noise and non-classical light are equal then $\gamma$ is equal to zero. The value of $\gamma$ is positive while the intensity of the noise is greater than the intensity of nonclassical light.

The joint distribution functions $P_{s i}^{N}\left(c_{\mathrm{s}}, c_{\mathrm{i}}\right)$ of the mixture parametric fluorescence $\left(\langle n\rangle=50, M_{s i}=20\right.$, Fig.3.a)) and thermal noise field $\left(\left\langle n_{s}\right\rangle=\left\langle n_{i}\right\rangle=50, M=20\right.$, Fig.3.b) ) are depicted in Fig.4.b). The additional uniform noise $\left(\left\langle n_{s}\right\rangle=\left\langle n_{i}\right\rangle=50, M=20\right)$ is able to erase the non-classical signature of parametric fluorescence $\left(\langle n\rangle=50, M_{s i}=20\right)$, even for perfect detectors $\left(T_{s}=T_{i}=1\right)$, as we can see from Table 1. (column title $P_{s i}{ }^{N}$ ). We obtain the same result if the convolution (16), Table 1., column title $P_{\eta, s i}{ }^{N}$, is accounted for a mixture of the same sources as in the previous case, except both sources are now independently affected by the same losses before detection $\left(T_{s}=T_{i}=0.2\right.$, $\left\langle n_{s}\right\rangle=\left\langle n_{i}\right\rangle=\langle n\rangle=10$, distributions $P_{\eta, s i}\left(m_{s}, m_{i}\right), \quad P_{\eta}\left(n_{i}, n_{j}\right)$, Fig.3.c), Fig.3.d)).

The above described case can be viewed as an event of detection of non-classical light by real detectors $\left(T_{s}=T_{i}=0.2\right)$ affected by the additional thermal noise $\left(\left\langle n_{s}\right\rangle=\left\langle n_{i}\right\rangle=10\right)$. The non-classical signature of the same field $\left(\langle n\rangle=50, M_{s i}=20\right)$ as before can now be erased by even a smaller amount of the noise $\left(\left\langle n_{s}\right\rangle=\left\langle n_{i}\right\rangle=10\right)$, while the low quantum efficiencies of detectors are accounted for. It is a crucial finding. The non-classical signature of the field is not affected by the drop of quantum efficiency of real noisy detectors if the dark-current noise level is also proportionally decreased. Otherwise, the non-classicality vanishes due to noise.

The thermal noise is usually hugely multimode $M \sim 100$ in real detector devices, much more than we considered until now $(M=20)$. It is important to find the threshold noise level $\gamma_{0}^{l m}$ in real condition $(M=100)$ at which the factor $R_{a b}(l, m)$ becomes zero. It is much easier to solve this problem if the relations between the factor $R_{a b}(l, m)$ and the noise level $\gamma$ are known. The four examples of such dependencies are depicted in Fig.4.d). The black and grey lines are accounted for the correlated photon pair source with $\langle n\rangle=50$ and $\langle n\rangle=15$. The dashed and solid lines correspond to number of modes $M_{s i}=1$ and $M_{s i}=20$. The dependencies are not affected by the quantum efficiency of the detectors. The circle $\left(\langle n\rangle=50, M_{s i}=20\right)$ and diamond $\left(\langle n\rangle=15, M_{s i}=20\right)$ markers accounted for the $T=0.2$, Fig.4.d) share the same curves as those accounted for the ideal detectors, $T=1$ (solid lines). While the noise signal is weak $\left(\gamma^{l m}<\gamma_{0}^{l m}\right)$, the impact of noise on non-classical signature field varies with the number of modes, even when the strength of the non-classical field remains the same. Lee's factor $R_{a b}(l, m)$ of the mixture is independent of the number of modes $M_{s i}$ for noise intensities higher than the threshold $\left(\gamma^{l m} \geq \gamma_{0}^{l m}\right)$. In the limit for $\gamma^{l m}>>\gamma_{0}^{l m}$, the factor $R_{a b}$ reaches the value corresponding to classical signal (dashed dot line, $M=100$, Fig.4.d)). The weak non-classical light requires a higher threshold noise level $\gamma_{0}^{l m}$ than the stronger one. The opposite is true if the relative noise level $\gamma_{0}^{l m}(17)$ is recalculated in absolute intensities of photon numbers $n_{0}{ }^{l m}$, Table 2. The threshold noise level $\gamma_{0}{ }^{11}$ has significantly suppressed the dependency on the number of modes $M_{s i}$, especially for weak intensities of non-classical signal, $\langle n\rangle<20$, Fig.4.e). The threshold levels $\gamma_{0}^{l m}$ of higher orders are bigger for non-classical fields of a few modes $M_{s i}<15$, as compared to highly multimode fields, Fig.4.f).

Unequally distributed noise, $\left\langle n_{s}\right\rangle \neq\left\langle n_{i}\right\rangle$, erases the nonclassical signature even for smaller noise level intensities as compared to that for uniformly distributed noise, Fig.4.c). The dashed line in Fig.4.c) delimits the area with negative factor $R_{a b}(1,1)$, the solid line delimits the area with negative $R_{a b}(4,4)$. The factor $R_{a b}(1,1)$ is observed to increase with intensity difference of noise channels. An additional increase of 9 photons of noise intensity in one arm can delete the non-classical signature for any initial intensity of 
the balanced noise beams, Fig.4.c). The balanced noise beams with higher intensities are much more sensitive to noise inhomogeneity as compared to the beams with lower intensities, dashed and solid lines, Fig.4.c). The higher orders of $R_{a b}(l, m)$ are a little bit less influenced by unbalanced noise beams, solid line, Fig.4.c).

Table 2. The relative $\gamma_{0}^{l m}$ and absolute $n_{0}{ }^{l m}$ threshold levels of mixed non-classical light and thermal noise $(M=100)$, Fig.4.d).

\begin{tabular}{|c|c|c|c|c|c|c|c|c|}
\hline$N_{s i}$ & $M_{s i}$ & $\gamma_{0}{ }^{11}$ & $\gamma_{0}^{21}$ & $\gamma_{0}^{22}$ & $\gamma_{0}^{31}$ & $\gamma_{0}^{32}$ & $\gamma_{0}^{33}$ & $\gamma_{0}^{44}$ \\
\hline \multirow{2}{*}{50} & 1 & 0.44 & 0.65 & 0.86 & 0.85 & 1.05 & 1.21 & 1.45 \\
\hline & 20 & 0.41 & 0.42 & 0.43 & 0.43 & 0.44 & 0.45 & 0.47 \\
\hline \multirow{2}{*}{15} & 1 & 1.58 & 1.86 & 2.21 & 2.17 & 2.52 & 2.88 & 3.53 \\
\hline & 20 & 1.58 & 1.56 & 1.60 & 1.56 & 1.58 & 1.62 & 1.64 \\
\hline$N_{s i}$ & $M_{s i}$ & $n_{0}{ }^{11}$ & $n_{0}^{21}$ & $n_{0}^{22}$ & $n_{0}^{31}$ & $n_{0}^{32}$ & $n_{0}^{33}$ & $n_{0}^{44}$ \\
\hline \multirow{2}{*}{50} & 1 & 72.0 & 82.6 & 93.4 & 92.9 & 102 & 110 & 122 \\
\hline & 20 & 70.7 & 71.1 & 71.7 & 71.6 & 72.1 & 72.8 & 73.9 \\
\hline \multirow{2}{*}{15} & 1 & 38.7 & 42.9 & 48.2 & 47.6 & 52.9 & 58.3 & 67.9 \\
\hline & 20 & 38.7 & 38.4 & 39.0 & 38.5 & 38.7 & 39.3 & 39.6 \\
\hline
\end{tabular}
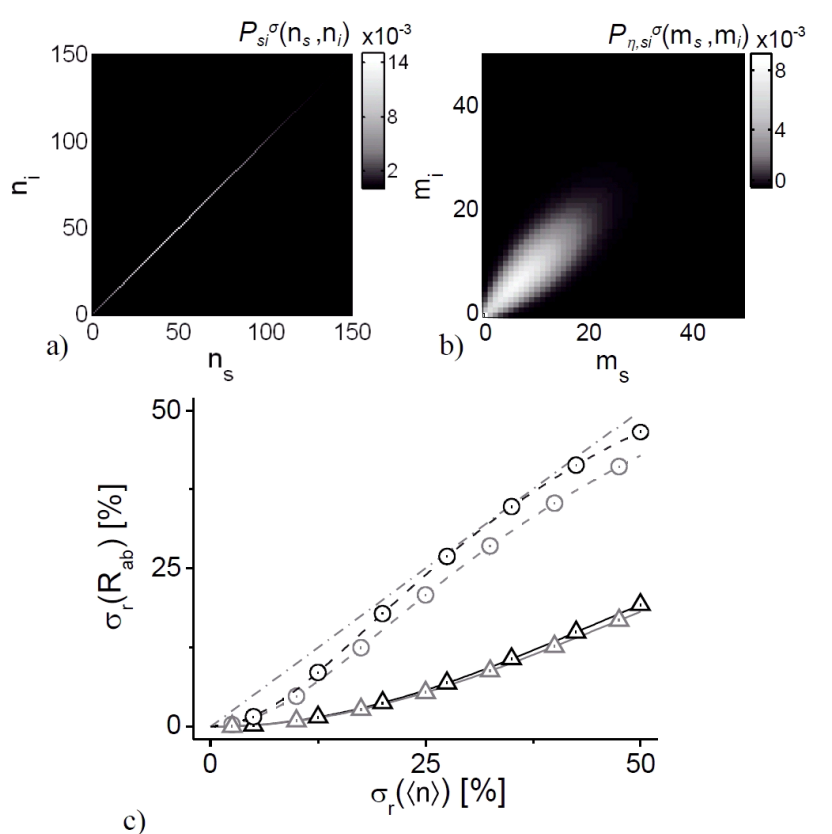

c)

Fig.5. Joint photon number distribution of non-classical signal, $\langle n\rangle=50, M_{s i}=20$ detected by a) ideal $T=1$, and b) real $T=0.2$ detector, generated by fluctuated pumping, $n_{\mu}=\langle n\rangle, \sigma_{r}(\langle n\rangle)=$ $50 \%$. c) The dependencies of relative deviation $\sigma_{r}\left(R_{a b}(1,1)\right)$ (solid lines) and $\sigma_{r}\left(R_{a b}(4,4)\right)$ (dashed lines) of non-classical light $(\langle n\rangle=50, M=20, T=1$, black color, $\langle n\rangle=10, M=20, T=1$, gray color) on size of relative standard deviation $\sigma_{r}(\langle n\rangle)$ of pumping intensity fluctuation $n_{\mu}=\langle n\rangle$. The markers correspond to real detectors, $T=0.2$.

The last parameter that reasonably influenced the statistics of the measured joint photon number distribution is the fluctuation of the pumping beam intensity. The fluctuations of pumping beam are mostly governed by the normal
Gaussian distribution. Under this consideration, the average number of photon pairs $\langle n\rangle$ (9) starts to fluctuate also. The generating function $P\left(n_{j}, M\right)$ (10) of joint distribution function $P_{s i}\left(n_{s}, n_{i}\right)$ (9) is no more defined by the negative binomial distribution and has to be calculated by the formula

$$
P^{\sigma}\left(n, M_{s i}\right)=\int_{-\infty}^{\infty} \frac{1}{\sigma(\langle n\rangle) \sqrt{2 \pi}} e^{\frac{-\left(\langle n\rangle-n_{\mu}\right)^{2}}{2 \sigma(\langle n\rangle)}} P\left(n, M_{s i}\right) d\langle n\rangle,
$$

where $n_{\mu}$ and $\sigma(\langle n\rangle)$ are the average value and standard deviation of photon pair number $\langle n\rangle$ affected by fluctuated pumping.

We are interested in the impact of the intensity fluctuation to factor $R_{a b}(l, m)$. For this reason, we introduce the relative standard deviation $\sigma_{r}(\langle n\rangle)$ of the fluctuating average photon pair number $\langle n\rangle$

$$
\sigma_{r}(\langle n\rangle)=100 \frac{\langle n\rangle}{\sigma(\langle n\rangle)}
$$

The modified joint distributions $P_{s i}{ }^{\sigma}, P_{\eta, s i}{ }^{\sigma}(9),(10),(12)$, of parametric fluorescence detected by ideal $(T=1$, Fig.3.a)) and real ( $T=0.2$, Fig.3.c)) detector are depicted in Fig.5.a) and Fig.5.b). The non-classical signature of distributions $P_{s i}{ }^{\sigma}, P_{\eta, s i}{ }^{\sigma}$ is still significant, moreover, the global statistics was changed, Table 1 . We considered the relative deviation $\sigma_{r}\left(R_{a b}\right)$ to monitor the changes in non-classical signature

$$
\sigma_{r}\left(R_{a b}\right)=100\left|\frac{R_{a b}^{\sigma}(l, m)-R_{a b}(l, m)}{R_{a b}(l, m)}\right|,
$$

where $R_{a b}^{\sigma}(l, m)$ and $R_{a b}(l, m)$ are Lee's factors (6) of the light fields generated by the fluctuating and constant pumping. The non-classical signature is influenced just marginally by small fluctuation of pump power $\sigma_{r}(\langle n\rangle)<1 \%$, as we can see from the dependence of $\sigma_{r}\left(R_{a b}\right)$ on the relative standard deviation of $\sigma_{r}(\langle n\rangle)$, Fig.5.c). The higher orders of non-classicality factor $R_{a b}$ (dashed lines in Fig.5.c)) increase nearly directly proportionally (dashed dot line) with size of pumping intensity fluctuation $\sigma_{r}(\langle n\rangle)$. The intensive parametric fluorescence (black lines in Fig.5.c)) is more sensitive to the pumping fluctuation than the weaker parametric fluorescence (grey lines in Fig.5.c)). The detector efficiency $(T=0.2$, circle and triangular markers in Fig.5.c)), does not modify the dependencies.

\section{EXPERIMENT}

The theoretical findings were used for the measurement of the quantum efficiency of the CCD cameras with image intensifiers. As the source of non-classical light we used the parametric fluorescence as was suggested in the theory. The 
photon pairs were generated in nonlinear BBO crystal of length $5 \mathrm{~mm}$, pumped by the third harmonics pulsed of Ti:sapphire laser system. The pulse width of laser oscillator was $200 \mathrm{fs}$ and repetition rate was $50 \mathrm{kHz}$. The central wavelength of laser oscillator was $840 \mathrm{~nm}$. The central wavelength of the third harmonics was $280 \mathrm{~nm}$. The pulse energy of the third harmonics was $45 \mathrm{~nJ}$. We were able to generate tens of photon pairs per pulse by this system. The wavelength of detected pairs was about $560 \mathrm{~nm}$. The vertex half angle of the cone was 12 degrees. Two regions of the cones, situated opposite to each other, were selected for the detection (picture placed to the left in Fig.6.d)). The first one - idler part - was reflected to the detector by high reflective mirror. The second one - signal part - was impinged to the detector directly. The length of the signal arm was $13 \mathrm{~cm}$. We used the CCD camera with image intensifiers (ICCD) Andor iStar DH 712-18-F-63 as the detector in our experimental setup. We divided the detection plane into three sections, Fig.6.d). The first one and the second one detected the idler and signal photons. The third one was used for estimating the noise level. The balanced detection of the signal and idler photons was achieved by detection of photons emitted into both arms with the same detector (ICCD camera). The imperfections of balancing are caused by asymmetrical detection scheme. To speed up the data collection, we decreased the resolution by grouping $8 \times 8$ pixels into one superpixel in the hardware of the camera. We placed the narrowband filter with bandwidth $14 \mathrm{~nm}$ (FWHM) in front of the camera to block parasite noise photons. The central wavelength of the filter was $560 \mathrm{~nm}$. We focused our attention mainly on the detection of degenerate parametric fluorescence. The measurement took typically several hours. For this reason we actively stabilized the power of pumping beam by feedback loop and polarization attenuator. The rate of stabilized pump power fluctuation did not exceed $0.3 \% \mathrm{rms}$. The exposure time of ICCD camera was set by the time interval between switch on and switch off of the camera intensifier. We achieved the best results for exposure time $7 \mathrm{~ns}$. The moment of switching on was synchronized with the pulse of the pump laser.

We usually obtained thousands of images at the end of the measurements (picture placed to the right in Fig.6.d)). The detection of photon corresponds to the white place of the image. We were interested in the rate of correlations of detection of both signal and idler photons. We selected the images with the same number combination of detection events $c_{s}, c_{i}$ in the signal and idler detection section and subsequently we counted the overall frequency $f\left(c_{s}, c_{i}\right)$ of these images according to the relation,

$$
f\left(c_{s}, c_{i}\right)=\frac{p\left(c_{s}, c_{i}\right)}{K},
$$

where $p\left(c_{s}, c_{i}\right)$ is the number of selected images and $K$ is the total number of all images. In the case of the source emitting the same number of photons into both arms, the overall frequency $f\left(c_{s}, c_{i}\right)$ has only nonzero diagonal components, whereas the other components are equal to zero. The overall frequency $f\left(c_{s}, c_{i}\right)$ counted from 1 million images is depicted in Fig.6.e). The statistics of the measured distribution $f\left(c_{s}, c_{i}\right)$ is summarized in Table 3 . The statistics of detection of events per strip is Super-Poissonian. Except for the nonclassicality factor $R_{a b}(4,4)$, in all other cases $f\left(c_{s}, c_{i}\right)$ are found to be negative, Table 3 .
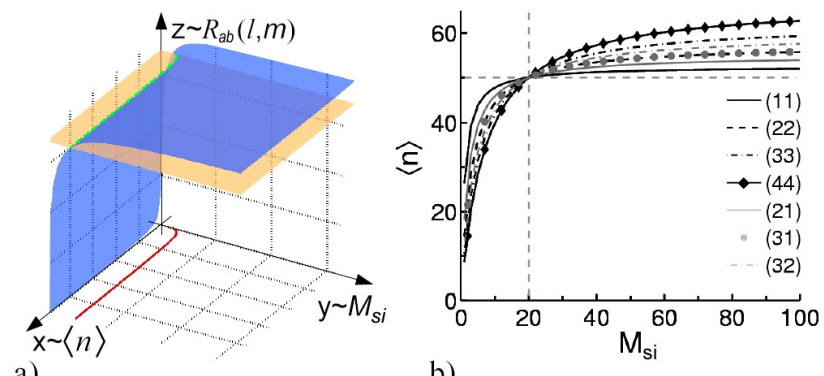

a)

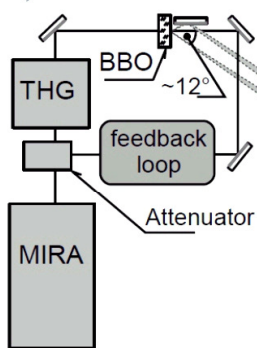

c)

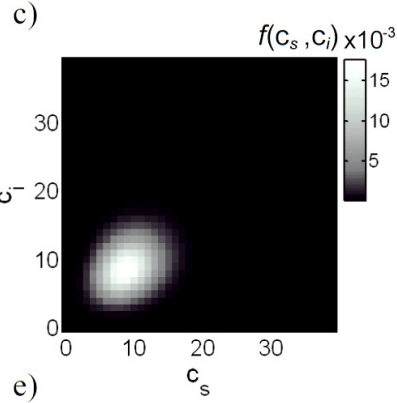

b)

d)

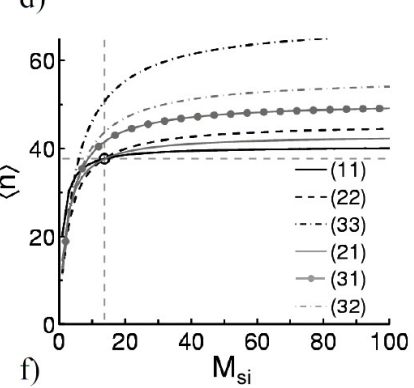

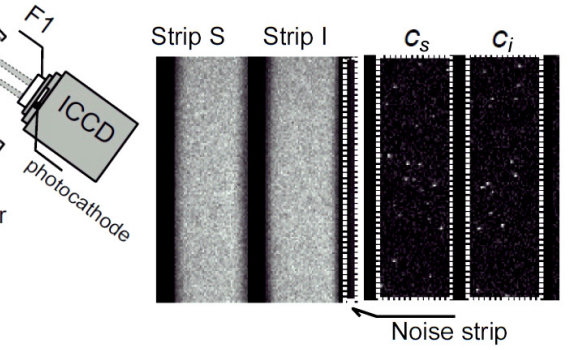

Fig.6. a). The geometrical interpretation of solving two equations for two unknowns. b) The graphical solution of the numerical procedure of finding average photon number $\langle n\rangle$ from the knowledge of the $R_{a b}(l, m)$, accounted for parametric fluorescence $\langle n\rangle=50, \quad M=20, \quad T=1$. c) Experimental setup used for the measurement of photon statistics of parametric fluorescence. d) The image with registered photons after illumination by parametric fluorescence coming from 10000 consecutive pump pulses (left) one pump pulse (right). e) Measured overall frequency $f\left(c_{s}, c_{i}\right)$. f) The graphical solution of the numerical procedure of finding average photon number $\langle n\rangle$ accounted for experimental data.

The graphical solution of the numerical procedure of finding average photon number $\langle n\rangle$ accounted for the experimental data (Table 3.) is depicted in Fig.6.f). The intersection points on the projected curves accounted for three orders $(1,1),(2,1),(2,2)$ are placed close to each other. The other intersection points are spread over a large area. This is caused by the slightly unbalanced transmittance of signal and idler arms together with the non-uniform spreading of the noise in the area of the CCD detector. The average value $\langle n\rangle$ of photon pairs was $\langle n\rangle=37.7 \pm 0.2$ and the number of modes $\left\langle M_{s i}\right\rangle=13.7 \pm 0.5$. The quantum efficiency (14) for both signal and idler detection section 
was $\left\langle\eta_{s}\right\rangle=(24.2 \pm 0.2) \%$ and $\left\langle\eta_{i}\right\rangle=(24.7 \pm 0.2) \%$. All resultant values of $\langle n\rangle, M_{\mathrm{si}}, \eta_{s}, \eta_{i}$ were expressed by average values of coordinates of three closest intersection points and appropriate standard deviations. We got the same values of the quantum efficiencies $\eta_{s}, \eta_{i}$ as in our previous work [12].

Table 3. The statistical parameters accounted for the overall frequency $f\left(c_{s}, c_{i}\right)$ depicted on Fig.6.e).

\begin{tabular}{lc}
\hline \hline & $f\left(c_{s}, c_{i}\right)$ \\
\hline$\sigma_{r}(\langle n\rangle)[\%]$ & 0.3 \\
$\left\langle n_{\eta, s}\right\rangle$ & 9.3057 \\
$\left\langle n_{\eta, i}\right\rangle$ & 9.1270 \\
$F_{s}$ & 1.0171 \\
$F_{i}$ & 1.0217 \\
$R_{a b}(1,1)$ & -0.024122 \\
$R_{a b}(2,1)$ & -0.044322 \\
$R_{a b}(2,2)$ & -0.020925 \\
$R_{a b}(3,1)$ & -0.055950 \\
$R_{a b}(3,2)$ & -0.055950 \\
$R_{a b}(3,3)$ & -0.033796 \\
$R_{a b}(4,4)$ & 0.000462 \\
$G_{12}$ & 1.027100 \\
\hline \hline
\end{tabular}

\section{DISCUSSION}

We presented the model of detection of classical and nonclassical light. We tested the parametric fluorescence for one of Lee's criteria of non-classicality. We show that the criterion is able to assess the non-classicality of the multimode field detected with only two sensors. The nonclassical signature of weak multimode parametric fluorescence is stronger than an intensive single mode one. Surprisingly, the non-classical signature of the field is not affected by the quantum efficiencies of balanced detectors, $\eta_{s}=\eta_{i}<100 \%$. Unfortunately, the relatively small unbalancing of the detector efficiencies vanishes the nonclassical signature. The non-classical light is much more resistant to thermal noise of the detectors, even for unequal spreading. The intensive and single mode non-classical light is more resistant to noise than the weak and single mode. The intensity fluctuation of the parametric fluorescence influences mostly the higher orders of non-classicality.

\section{CONCLUSION}

We showed two possible applications of the non-classical light in metrology. The value of both the light irradiance and the quantum efficiency of detectors could be determined by the measurement of non-classical signature of the field. We used this method for measurement of the quantum efficiency of CCD camera with image intensifier. The method can be applied to detectors with low dark-current noise only. The sensitivity to noise increases as the detector quantum efficiency decreases. At present the method is developed only for balanced detectors with equal dark-current noise. In the future we will try to generalize the method for unbalanced detectors, too.

\section{ACKNOWLEDGMENT}

We acknowledge the support by the project P205/12/0382 of the Czech Science Foundation, by the Operational Program Research and Development for Innovations European Regional Development Fund (project CZ.1.05/2.1.00/03.0058) and Operational Program Education for Competitiveness - European Social Fund (project CZ.1.07/2.3.00/20.0017). A.P. also thanks the department of Science and Technology (DST), India for support provided thorough the DST project no. SR/S2/LOP$0012 / 2010$

\section{REFERENCES}

[1] Yurke, B., Whittaker, E.A. (1987). Squeezed state enhanced frequency-modulation spectroscopy. Optics Letters, 12 (4), 236-238.

[2] Xiao, M., Wu, L.A., Kimble, H.J. (1987). Precision measurement beyond the shot-noise limit. Physical Review Letters, 59 (3), 278-281.

[3] Caves, C.M. (1981). Quantum-mechanical noise in an interferometer. Physical Review D, 23 (8), 1693-1708.

[4] Teich, M.C., Saleh, B.E.A. (1988). Photon bunching and antibunching. In Wolf, E. (ed.) Progress in Optics. Elsevier, Vol. 26, 1-104.

[5] Saleh, B.E.A., Teich, M.C. (1987). Can the channel capacity of a light-wave communication system be increased by the use of photon-number-squeezed light? Physical Review Letters, 58 (25), 2656-2659.

[6] Yamazaki, K., Hirota, O., Nakagawa, M. (1988). Optimum condition of squeezed state signal for optical communication with energy loss. Transactions of the Institute of Electronics, Information and Communication Engineers E, 71 (8), 775-783.

[7] Teich, M.C., Prucnal, P.R., Vannucci, G., Breton, M.E., McGill, W.J. (1982). Multiplication noise in the human visual system at threshold. Biological Cybernetics, 44, 157-165.

[8] Migdall, A.L., Datla, R.U., Sergienko, A., Orszak, J.S., Shih, Y.H. (1995/96). Absolute detektor quantum-efficiency measurement ussing correlated photons. Metrologia, 32 (6), 479-483.

[9] Brida, G., Degiovanni, I.P., Genovese, M., Rastello, M.L., Ruo-Berchera, I. (2010). Detection of multimode spatial correlation in PDC and application to the absolute calibration of a CCD camera. Optics Express, 18 (20), 20572.

[10] Polyakov, S.V., Wareb, M., Migdalla, A. (2006). High accuracy calibration of photon-counting detectors. In Photon Counting Applications, Quantum Optics, and Quantum Cryptography, Proc. of SPIE 6372.

[11] Polyakov, S.V., Migdall, A.L. (2007). High accuracy verification of a correlatedphoton-based method for determining photoncounting detection efficiency. Optics Express, 15 (4), 1390-1407.

[12] Peřina, J. Jr., Haderka, O., Michálek, V., Hamar, M. (2012). Absolute detector calibration using twin beams. Optics Letters, 37 (13), 2475-2477. 
[13] Loundon, R., Knight, B.L. (1987). Squeezed light. Journal of Modern Optics, 34 (6-7), 709-759.

[14] Haderka, O., Peřina, J. Jr., Hamar, M., Peřina, J. (2005). Direct measurement and reconstruction of nonclassical features of twin beams generated in spontaneous parametric down-conversion. Physical Review A, 71 (3), 033815-033819.

[15] Waks, E., Sanders, B.C., Diamanti, E., Yamamoto, Y. (2006). Highly non-classical photon statistics in parametric down-conversion. Physical Review A, 73 (3), 033814.

[16] Waks, E., Diamanti, E., Sanders, B.C., Bartlett, S.D., Yamamoto, Y. (2004). Direct observation of nonclassical photon statistics in parametric downconversion. Physical Review Letters, 92 (11), 113602.

[17] Haderka, O., Hamar, M., Peřina, J. Jr. (2004). Experimental multi-photon-resolving detector using a single avalanche photodiode. European Physical Journal D, 28 (1), 149-154.

[18] Haderka, O., Peřina, J. Jr., Hamar, M. (2005). Simple direct measurement of non-classical joint signal-idler photon-number statistics and the correlation area of twin photon beams. Journal of Optics B: Quantum and Semiclassical Optics, 7 (12), S572-S576.

[19] Breitenbach, G., Schiller, S., Mlynek, J. (1997). Measurement of the quantum states of squeezed light. Nature, 387 (6632), 471-475.

[20] Peřina, J. Jr., Haderka, O., Michálek, V., Hamar, M. (2013). State reconstruction of a multimode twin beam using photodetection. Physical Review A, 87 (2), 022108-022113.

[21] Hillery, M. (1985). Conservation laws and nonclassical states in nonlinear optical systems. Physical Review A, 31 (1), 338-342.

[22] Hillery, M. (1989). Total noise and non-classical states. Physical Review A, 39 (6), 2994-3002.
[23] Lee, C.T. (1990). Higher-order criteria for nonclassical effects in photon statistics. Physical Review A, 41 (3), 1721-1723.

[24] Lee, C.T. (1998). Simple criterion for non-classical two-mode states. Journal of the Optical Society of America B, 15 (3), 1187-1191.

[25] Lee, C.T. (1990). Many-photon antibunching in generalized pair coherent states. Physical Review A, 41 (3), 1569-1575.

[26] Lee, C.T. (1990). General criteria for non-classical photon statistics in multimode radiations. Optics Letters, 15 (23), 1386-1388.

[27] Agarwal, G.S. (1988). Generation of squeezed states of radiation at submultiple Rabi resonances. Journal of the Optical Society of America B, 5 (1), 180-183.

[28] Hong, C.K., Mandel, L. (1985). Higher-order squeezing of a quantum field. Physical Review Letters, 54 (4), 323-325.

[29] Gerry, Ch., Knight, P. (2005). Introductory Quantum Optics. Cambridge University Press.

[30] Scully, M.O., Zubairy, M.S. (1997). Quantum Optics. Cambridge University Press.

[31] Hamar, M., Peřina, J. Jr., Haderka, O., Michálek, V. (2010). Transverse coherence of photon pairs generated in spontaneous parametric down-conversion. Physical Review A, 81 (4), 043827-043836.

[32] Peřina, J., Křepelka, J. (2005). Multimode description of spontaneous parametric down-conversion. Journal of Optics B: Quantum and Semiclassical Optics, 7, 246-252.

[33] Allevi, A., Olivares, S., Bondani, M. (2012). Measuring high-order photon-number correlations in experiments with multimode pulsed quantum states. Physical Review A, 85 (6), 063835. 